UDC 548.735:[546.732+547.486]-386

\author{
E.K. Trunova, A.M. Mishchenko, A.A. Rogovtsov, T.A. Makotryk
}

\title{
CRYSTAL STRUCTURE OF A NOVEL COBALT(II) COMPLEX WITH ETHYLENEDIAMINEDISUCCINIC ACID
}

\author{
V.I. Vernadsky Institute of General and Inorganic Chemistry of the NAS of Ukraine, Kyiv
}

\begin{abstract}
Due to their valuable properties, coordination compounds of $3 \mathrm{~d}$ transition metals with aminocarboxylic acids are perspective bioactive compounds. In the present work, potassium (ethylenediamine-N,N'-disuccinato)cobaltate(II) dihydrate was prepared and structurally characterized by single-crystal X-ray diffraction analysis. Structure determination was performed by a least-squares fit of 6669 reflections of highest intensity and refined with the full-matrix least squares procedure. Present compound is a closed-type binuclear $\mathrm{Co}(\mathrm{II})$ complex of ethylenediaminedisuccinic acid composed of $\left[\left(\mathrm{C}_{10} \mathrm{H}_{12} \mathrm{~N}_{2} \mathrm{O}_{8}\right)_{2} \mathrm{Co}_{2}\right]$ moieties bridged via potassium atoms in a zig-zag fashion and possesses the monoclinic $\mathrm{P} 2_{1} / \mathrm{c}$ space group. Each $\mathrm{Co}$ (II) ion has a distorted octahedral environment formed by sets of three 5-membered and one 6-membered chelate cycles. One of the positions in the $\left[\mathrm{CoO}_{4} \mathrm{~N}_{2}\right]$ octahedron is occupied by oxygen atom of the neighboring ligand molecule. The acetate branches of ligand are situated in the axial positions of the distorted $\mathrm{Co}$ (II) octahedron on the contrary to previously studied sodium-containing complex of analogous composition. Each potassium atom has a seven-fold coordination involving three oxygen atoms of water molecules, two oxygen atoms from the nearest carboxyl group and two additional oxygen atoms from two adjacent ligand molecules. The complex under study also displays a developed system of inter-ligand $\mathrm{N}-\mathrm{H} \cdots \mathrm{O}, \mathrm{C}-\mathrm{H} \cdots \mathrm{O}$ and hydration $\mathrm{O}-\mathrm{H} \cdots \mathrm{O}$ bonds.
\end{abstract}

Keywords: cobalt complex, ethylenediaminedisuccinic acid, X-ray structure analysis, chelate cycle, hydrogen bond.

DOI: $10.32434 / 0321-4095-2018-121-6-84-90$

\section{Introduction}

The coordination compounds of $3 \mathrm{~d}$ metals with aminocarboxylic acids are perspective bioactive compounds due to their high stability, water solubility and, in consequence, high assimilability by living organisms. Ethylenediaminedisuccinic acid (EDDS, commonly denoted as $\mathrm{H}_{4}$ edds to emphasize its tetrabasic character) is a biodegradable chelating agent that can be used as a substitute for ethylenediaminetetraacetic acid in different applications, e.g. for the extraction of heavy metals from aqueous solutions, soils and phytoextraction [1-3], in the pulp and paper-making industry and for radionuclide decontamination [4]. The complexes of biogenic metals $\left(\mathrm{Mn}^{\mathrm{II}}, \mathrm{Fe}^{\mathrm{III}}, \mathrm{Co}^{\mathrm{II}}, \mathrm{Cu}^{\mathrm{II}}, \mathrm{Zn}^{\mathrm{II}}\right)$ with EDDS have attracted much interest due to their potential and practical applications in biochemistry $[5,6]$. Since its molecule contains two natural fragments (the residues of succinic and aspartic acids), not only does EDDS transport microelement but it also acts as a biostimulating component.

Among other microelements, cobalt is responsible for accumulation of sugars and lipids in plants and favors the synthesis of chlorophyll. As far as we know, only a few Co-based monometallic complexes with EDDS were structurally characterized previously [7-9]. Recently we have reported the structure of polymeric complex in which dimeric $\left[\mathrm{Co}_{2}(\mathrm{edds})_{2}\right]^{4-}$ units are connected via outersphere $\mathrm{Na}^{+}$counterions [10]. Each $\mathrm{Co}(\mathrm{II})$ ion has a distorted octahedral $\left[\mathrm{CoO}_{4} \mathrm{~N}_{2}\right]$ environment formed by sets of three 5-membered and one 6-membered chelate cycles. Sodium ions have a distorted trigonalbipyramidal coordination environment defined by three equatorial oxygen atoms of $\alpha$-carboxylic groups and two coordinated water molecules in the axial position. To study the effect of the alkaline metal ion substitution on the spatial arrangement of chelate cycles, we have synthesized another Co(II) complex with EDDS containing potassium as counterion. In

(C) E.K. Trunova, A.M. Mishchenko, A.A. Rogovtsov, T.A. Makotryk, 2018 
the present work its structure was determined by single-crystal X-ray diffraction analysis and compared with sodium-containing complex.

\section{Experimental}

Materials and methods

EDDS was prepared by condensation reaction of maleic acid with ethylenediamine [11]. All other chemicals were analytical grade and used as received.

Elemental analysis for carbon, hydrogen and nitrogen was performed by the combustion method with a Perkin-Elmer CHN 2400 analyzer. The metal contents were determined by atomic absorption using a Philips Pye Unicam 8000 atomic absorption spectrophotometer. IR spectrum was recorded on a Specord M80 spectrophotometer (Carl Zeiss Jena) as $\mathrm{KBr}$ pellet in the $400-4000 \mathrm{~cm}^{-1}$ range.

\section{Preparation}

A mixture of $\mathrm{CoCl}_{2} \cdot 6 \mathrm{H}_{2} \mathrm{O}(2.38 \mathrm{~g}, 10 \mathrm{mmol})$ and EDDS (2.92 g, $10 \mathrm{mmol})$ was dissolved in distilled water $(10 \mathrm{ml})$. Then $\mathrm{pH}$ was adjusted to 5.0 by concentrated $(40 \%)$ solution of $\mathrm{KOH}$. Reaction mixture was refluxed under constant stirring for $24 \mathrm{~h}$. After cooling to room temperature, diethyl ether was added into the solution giving a powder crude product. Precipitate was filtered off and washed with methanol for several times (yield 83\%). Anal. Calcd for $\mathrm{K}_{2}\left[\mathrm{Co}\right.$ (edss)] $2 \mathrm{H}_{2} \mathrm{O}$ (\%): K 16.9; Co 12.8; C 26.0; H 3.5; N 6.1. Found: K 16.6; Co 13.0; C 26.1; H 3.4; $\mathrm{N}$ 6.3. IR $\left(\mathrm{cm}^{-1}\right): 3460,3224\left(\mathrm{vH}_{2} \mathrm{O}\right) ; 2912,2364$ $(v \mathrm{C}-\mathrm{H}) ; 1650,1583\left(v_{\mathrm{as}} \mathrm{COO}^{-}\right) ; 1492,1390,1319$ $\left(v_{\mathrm{s}} \mathrm{COO}^{-}\right) ; 1060,1040,877(\mathrm{vC}-\mathrm{N}) ; 964,924(\mathrm{vC}-\mathrm{C})$; 708 (8OCO); 626, 603, $537(\mathrm{vCo}-\mathrm{O})$; $449(\mathrm{vCo}-\mathrm{N})$.

To obtain crystals suitable for $\mathrm{X}$-ray diffraction, the complex was dissolved in water and was stored in a dark place for slow evaporation. After 8 days violet prismatic crystals were formed.

\section{Crystallography}

Diffraction data were collected at 293(2) K by the $\varphi$ - and $\omega$-scan technique up to $2 \theta=60.5^{\circ}$, on a Bruker Smart Apex II with graphitemonochromatized MoKa radiation $(\lambda=0.71073 \AA)$. The data were corrected for Lorentz-polarization and absorption effects. Accurate unit-cell parameters were determined by a least-squares fit of 6669 reflections of highest intensity, chosen from the whole experiment. The structures were solved with DIRDIF-2008 program system and refined with the full-matrix least squares procedure on $\mathrm{F}^{2}$ by SHELXL97 [12]. Scattering factors incorporated in SHELXL97 were used. The function $\mathrm{R}_{\mathrm{w}}\left(\mathrm{F}_{\mathrm{o}}{ }^{2}-\mathrm{F}_{\mathrm{c}}{ }^{2}\right)^{2}$ was minimized with $\mathrm{w}^{-1}=\left[\sigma^{2}\left(\mathrm{~F}_{\mathrm{o}}\right)^{2}+(0.0209 \mathrm{P})^{2}+\right.$ $+0.6157 \mathrm{P}]$ (where $\mathrm{P}=\left[\max \left(\mathrm{F}_{\mathrm{o}}^{2}, 0\right)+2 \mathrm{~F}_{\mathrm{c}}^{2}\right] / 3$ ). All nonhydrogen atoms were refined anisotropically, hydrogen atoms were put in the idealized positions
Table 1

Crystal data, data collection and structure refinement

\begin{tabular}{|c|c|}
\hline Empirical formula & $\mathrm{C}_{10} \mathrm{H}_{16} \mathrm{CoN}_{2} \mathrm{~K}_{2} \mathrm{O}_{10}$ \\
\hline Formula weight $\left(\mathrm{g} \mathrm{mol}^{-1}\right)$ & 461.41 \\
\hline Temperature (K) & 293 \\
\hline Wavelength $(\AA)$ & 0.71073 \\
\hline Crystal system & Monoclinic \\
\hline Space group & $\mathrm{P} 2_{1} / \mathrm{c}$ \\
\hline $\mathrm{a}(\AA)$ & $7.8016(1)$ \\
\hline $\mathrm{b}(\AA)$ & $10.1712(2)$ \\
\hline $\mathrm{c}(\AA)$ & $18.5122(4)$ \\
\hline$\overline{\beta(0)}$ & $95.349(1)$ \\
\hline $\mathrm{V}\left(\AA^{3}\right)$ & $1462.58(5)$ \\
\hline $\mathrm{Z}$ & 4 \\
\hline $\mathrm{D}_{\text {Calcd }}\left(\mathrm{g} \mathrm{cm}^{-3}\right)$ & 1.918 \\
\hline$\mu\left(\mathrm{mm}^{-1}\right)$ & 1.52 \\
\hline $\mathrm{F}(000)$ & 864 \\
\hline Crystal size $(\mathrm{mm})$ & $0.1 \times 0.1 \times 0.1$ \\
\hline$\theta$ range $\left(^{\circ}\right)$ & $2.287-30.563$ \\
\hline Index ranges & $\begin{array}{c}-9 \leq \mathrm{h} \leq 9 \\
0 \leq \mathrm{k} \leq 12 \\
0 \leq 1 \leq 22 \\
\end{array}$ \\
\hline \multicolumn{2}{|l|}{ Reflections: } \\
\hline Collected & 6669 \\
\hline Unique $\left(\mathrm{R}_{\text {int }}\right)$ & $2875(0.03)$ \\
\hline With $\mathrm{I}>2 \sigma(\mathrm{I})$ & 2572 \\
\hline Number of parameters & 217 \\
\hline Final $\mathrm{R}_{1} / \mathrm{wR}_{2}[\mathrm{I}>2 \sigma(\mathrm{I})]$ & $0.0219 / 0.0567$ \\
\hline$\underline{\mathrm{R}_{1} / \mathrm{wR}_{2} \text { (all data) }}$ & $0.0258 / 0.0612$ \\
\hline $\operatorname{Max} / \min , \mathrm{e} / \AA^{3}$ & $0.37 /-0.21$ \\
\hline Goodness-of-fit on $\mathrm{F}^{2}$ & 1.05 \\
\hline
\end{tabular}

(those from water molecules and imines groups was found in the difference Fourier map), and refined as riding model. Their isotropic thermal parameters were set 1.2 times larger compared with the appropriate carrier atom. Relevant crystal data are listed in Table 1, together with refinement details. Table 2 contains atomic coordinates and displacement parameters.

\section{Results and discussion}

In contrast to previously reported sodiumcontaining orthorhombic Co(II)-EDDS complex [10], the obtained compound crystallizes in the monoclinic $\mathrm{P} 2_{1} / \mathrm{c}$ space group. Its asymmetric unit is shown in Fig. 1. Bond lengths and angles of slightly distorted $\left[\mathrm{CoO}_{4} \mathrm{~N}_{2}\right]$ octahedron (Table 3) fall in the ranges typical for octahedral cobalt complexes $[9$, 13].

Each cobalt atom is coordinated by N1, N2 atoms from ethylenediamine bridge and $\mathrm{O} 1, \mathrm{O} 3, \mathrm{O} 5$ 
Fractional atomic coordinates and isotropic or equivalent isotropic displacement parameters $\left(\mathrm{E}^{2}\right)$

\begin{tabular}{|c|c|c|c|c|}
\hline Atom & $\mathrm{x}$ & $\mathrm{y}$ & $\mathrm{z}$ & $\mathrm{U}_{\mathrm{iso}} * / \mathrm{U}_{\mathrm{eq}}$ \\
\hline Co1 & $0.05715(3)$ & $0.04342(2)$ & $0.362730(10)$ & $0.00930(10)$ \\
\hline K1 & $0.24325(5)$ & $0.45653(4)$ & $0.45811(2)$ & $0.01440(10)$ \\
\hline $\mathrm{O} 1$ & $0.06654(14)$ & $0.20908(11)$ & $0.41257(6)$ & $0.0124(3)$ \\
\hline $\mathrm{O} 2$ & $-0.09642(15)$ & $0.34518(12)$ & $0.47055(7)$ & $0.0181(4)$ \\
\hline $\mathrm{O} 3$ & $-0.06631(14)$ & $0.12167(11)$ & $0.28128(6)$ & $0.0128(3)$ \\
\hline $\mathrm{O} 4$ & $-0.11860(16)$ & $0.08758(12)$ & $0.16260(6)$ & $0.0180(4)$ \\
\hline $\mathrm{O} 5$ & $0.26779(14)$ & $0.07690(11)$ & $0.31920(6)$ & $0.0133(3)$ \\
\hline O6 & $0.45301(15)$ & $0.05250(12)$ & $0.23687(7)$ & $0.0187(4)$ \\
\hline O7 & $-0.16937(14)$ & $0.03242(11)$ & $0.55161(6)$ & $0.0132(3)$ \\
\hline O8 & $-0.34768(15)$ & $0.17135(12)$ & $0.60109(6)$ & $0.0165(4)$ \\
\hline O9 & $0.54576(16)$ & $0.60697(12)$ & $0.41856(7)$ & $0.0202(4)$ \\
\hline O10 & $0.40815(17)$ & $0.33412(13)$ & $0.35125(7)$ & $0.0254(4)$ \\
\hline N1 & $-0.15643(17)$ & $0.01962(13)$ & $0.40389(7)$ & $0.0109(4)$ \\
\hline $\mathrm{N} 2$ & $0.01538(17)$ & $-0.12104(13)$ & $0.31358(7)$ & $0.0116(4)$ \\
\hline $\mathrm{C} 1$ & $-0.0774(2)$ & $0.24386(16)$ & $0.43601(9)$ & $0.0128(5)$ \\
\hline $\mathrm{C} 2$ & $-0.2300(2)$ & $0.15161(17)$ & $0.41609(9)$ & $0.0120(5)$ \\
\hline $\mathrm{C} 3$ & $-0.2654(2)$ & $-0.07085(17)$ & $0.35624(9)$ & $0.0149(5)$ \\
\hline $\mathrm{C} 4$ & $-0.1471(2)$ & $-0.17954(17)$ & $0.33614(9)$ & $0.0150(5)$ \\
\hline $\mathrm{C} 5$ & $0.0151(2)$ & $-0.08531(17)$ & $0.23585(9)$ & $0.0132(5)$ \\
\hline C6 & $-0.0669(2)$ & $0.04957(16)$ & $0.22327(9)$ & $0.0125(5)$ \\
\hline $\mathrm{C} 7$ & $0.2026(2)$ & $-0.07714(17)$ & $0.21884(9)$ & $0.0152(5)$ \\
\hline $\mathrm{C} 8$ & $0.3152(2)$ & $0.02428(16)$ & $0.26106(9)$ & $0.0138(5)$ \\
\hline C9 & $-0.3627(2)$ & $0.15381(17)$ & $0.47154(9)$ & $0.0134(5)$ \\
\hline $\mathrm{C} 10$ & $-0.2902(2)$ & $0.11981(16)$ & $0.54829(9)$ & $0.0122(5)$ \\
\hline $\mathrm{H} 2$ & 0.25468 & -0.16295 & 0.22758 & 0.0182 \\
\hline $\mathrm{H} 3$ & 0.20398 & -0.0584 & 0.16752 & 0.0182 \\
\hline $\mathrm{H} 4$ & -0.04722 & -0.1514 & 0.20512 & 0.0158 \\
\hline $\mathrm{H} 5$ & 0.1016 & -0.17834 & 0.32417 & 0.0139 \\
\hline H6 & -0.1209 & -0.23731 & 0.37738 & 0.018 \\
\hline $\mathrm{H} 7$ & -0.20315 & -0.23107 & 0.29664 & 0.018 \\
\hline $\mathrm{H} 8$ & -0.31451 & -0.0252 & 0.31317 & 0.0179 \\
\hline H9 & -0.35836 & -0.10605 & 0.38176 & 0.0179 \\
\hline $\mathrm{H} 10$ & -0.13275 & -0.01371 & 0.44716 & 0.0131 \\
\hline H11 & -0.41372 & 0.24075 & 0.47175 & 0.0161 \\
\hline $\mathrm{H} 12$ & -0.45367 & 0.09196 & 0.45642 & 0.0161 \\
\hline H13 & -0.28747 & 0.18173 & 0.36972 & 0.0143 \\
\hline H14 & 0.53778 & 0.57518 & 0.38224 & 0.0303 \\
\hline $\mathrm{H} 15$ & 0.48712 & 0.68169 & 0.41084 & 0.0303 \\
\hline H16 & 0.38043 & 0.25671 & 0.33273 & 0.0381 \\
\hline H17 & 0.44406 & 0.37579 & 0.31431 & 0.0381 \\
\hline
\end{tabular}

atoms of three carboxylate groups and forms three 5-membered rings: $\mathrm{Co} 1-\mathrm{O} 3-\mathrm{C} 6-\mathrm{C} 5-\mathrm{N} 2$ $\left(\varphi=333.6(2)^{0}\right.$, twisted conformation), glycine cycle Co1-O1-C1-C2-N1 $\left(\varphi=322.3(2)^{0}\right.$, envelope conformation), ethylenediamine cycle $\mathrm{Col}-\mathrm{N} 1-$ $\mathrm{C} 3-\mathrm{C} 4-\mathrm{N} 2\left(\varphi=71.94(17)^{0}\right.$, envelope conformation), and one 6-membered $\beta$-alanine ring $\mathrm{Co} 1-\mathrm{O} 5-\mathrm{C} 8-$ C7-C5-N2 $\left(\tau=131.16(13)^{0}, \varphi=102.58(16)^{0}\right)[14]$. The sixth position in the $\left[\mathrm{CoO}_{4} \mathrm{~N}_{2}\right]$ octahedron is occupied by oxygen atom of the neighboring EDDS molecule $\left[\mathrm{d}\left(\mathrm{O} 7 \cdots \mathrm{O}^{\mathrm{i}}\right)=3.4658(16) \AA\right]$ producing a binuclear moiety (Fig. 2). The forth carboxylate group of EDDS is non-coordinated and possess outward direction coplanar with (110) plane. The glycine and ethylenediamine rings are situated in the equatorial plane of the $\left[\mathrm{CoO}_{4} \mathrm{~N}_{2}\right]$ octahedron forming a conjugated system, while the $\beta$-alanine ring is located perpendicularly. 
Table 3 Geometrical parameters of the $\left[\mathrm{CoO}_{4} \mathrm{~N}_{2}\right]$ polyhedron

\begin{tabular}{c|c|c|c}
\hline Bond & $\mathrm{d}, \AA$ & Angles & $\theta,^{0}$ \\
\hline Co1-O1 & $1.9192(11)$ & O1-Co1-O3 & $90.44(5)$ \\
\hline Co1-O3 & $1.8878(11)$ & O3-Co1-N2 & $86.41(5)$ \\
\hline Co1-O5 & $1.9269(11)$ & O1-Co1-N1 & $85.13(5)$ \\
\hline Co1-O7 & $1.9019(11)$ & O1-Co1-N2 & $172.40(5)$ \\
\hline Co1-N1 & $1.9111(13)$ & O1-Co1-O5 & $92.87(5)$ \\
\hline Co1-N2 & $1.9175(13)$ & N1-Co1-N2 & $87.87(6)$ \\
\hline
\end{tabular}

Apart from metal-ligand bonds, a number of intramolecular hydrogen bonds ranging from $2.7385(17)$ to $3.0920(18) \AA$ are presented in the complex (Table 4). Water molecules interact with $\mathrm{N} 2$ donor atom resulting in extended $\mathrm{N}-\mathrm{H} \cdots \mathrm{O}$ hydrogen bond network, while the sequence of $\mathrm{N} 1-\mathrm{H} 10 \cdots \mathrm{O} 7$ bonds displays chain-like arrangement (Fig. 3).

$\mathrm{K} 1$ atom coordinates three oxygen atoms $\mathrm{O} 9, \mathrm{O} 10$, $\mathrm{O}^{\mathrm{iv}}$ of water molecules $[\mathrm{d}(\mathrm{K} 1 \cdots \mathrm{O} 9)=2.9613(13) \AA$, $\left.\mathrm{d}(\mathrm{K} 1 \cdots \mathrm{O} 10)=2.7555(14) \AA, \mathrm{d}\left(\mathrm{K} 1 \cdots \mathrm{O}^{\mathrm{iv}}\right)=2.7638(13) \AA\right]$, two oxygen atoms $\mathrm{O} 1, \mathrm{O} 2$ from the same carboxyl group $[\mathrm{d}(\mathrm{K} 1 \cdots \mathrm{O} 1)=2.9545(12) \AA, \mathrm{d}(\mathrm{K} 1 \cdots \mathrm{O} 2)=2.9106(12) \AA]$ and two additional oxygens $\mathrm{O} 2^{\mathrm{iii}}$ and $\mathrm{O}^{\mathrm{ii}}$ from two

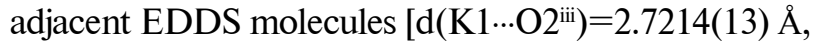
$\left.\mathrm{d}\left(\mathrm{K} 1 \cdots \mathrm{O} 4^{\mathrm{ii}}\right)=2.7038(12) \AA\right]$ (Fig. 1). Two nearest potassium atoms share common edge of their polyhedrons along the $\mathrm{O} 2 \cdots \mathrm{O} 2^{\mathrm{i}}$ direction forming a singular cell comprised of four $\left[\left(\mathrm{C}_{10} \mathrm{H}_{12} \mathrm{~N}_{2} \mathrm{O}_{8}\right)_{2} \mathrm{Co}_{2}\right]$ moieties with a volume of ca. $12.6 \times 17.3 \times 30.4 \AA^{3}$. Potassium bipolyhedral assemblies extend along the

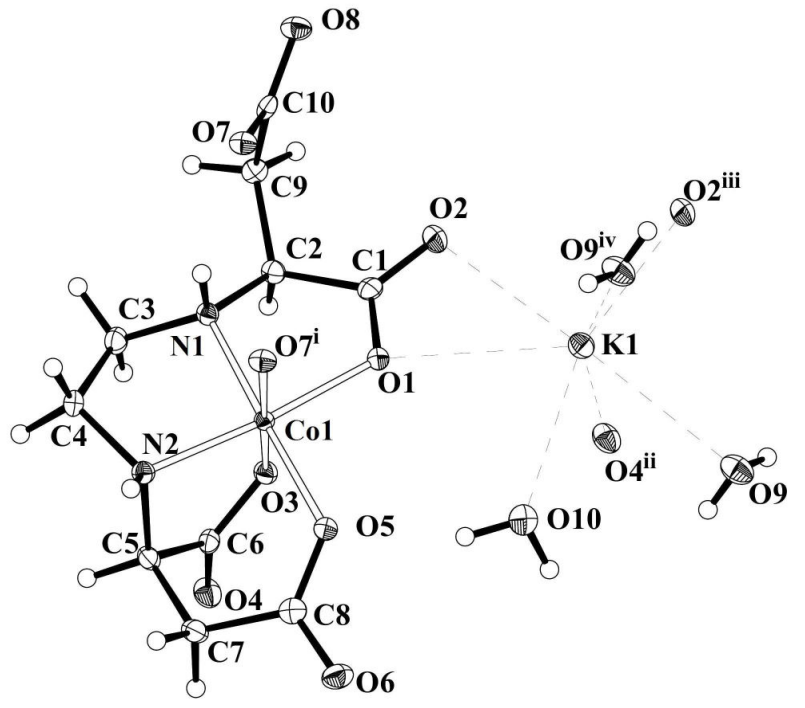

Fig. 1. Anisotropic ellipsoid representation of the complex asymmetric unit together with atom labeling scheme. The ellipsoids are drawn at $50 \%$ probability level, hydrogen atoms are depicted as spheres with arbitrary radii, $\mathrm{K} \cdots \mathrm{O}$ contacts are shown as the dashed lines. Symmetry codes:

$$
\text { (i) }-\mathrm{x},-\mathrm{y},-\mathrm{z}+1 \text {; (ii) }-\mathrm{x}, \mathrm{y}+\mathrm{S},-\mathrm{z}+\mathrm{S} \text {; }
$$

(iii) $-\mathrm{x},-\mathrm{y}+1,-\mathrm{z}+1$; (iv) $-\mathrm{x}+1,-\mathrm{y}+1,-\mathrm{z}+1$

[001] direction in a zig-zag fashion with a period of translation equal to the lattice constant $\mathrm{a}=7.8016(1) \AA$.

\section{Conclusions}

To sum up, single-crystal X-ray diffraction analysis revealed a structural rearrangement which takes place in a $\mathrm{Co}(\mathrm{II})-\mathrm{EDDS}$ monometallic complex when the $\mathrm{Na}^{+}$counterion is replaced with $\mathrm{K}^{+}$. In the potassium-containing complex the acetate

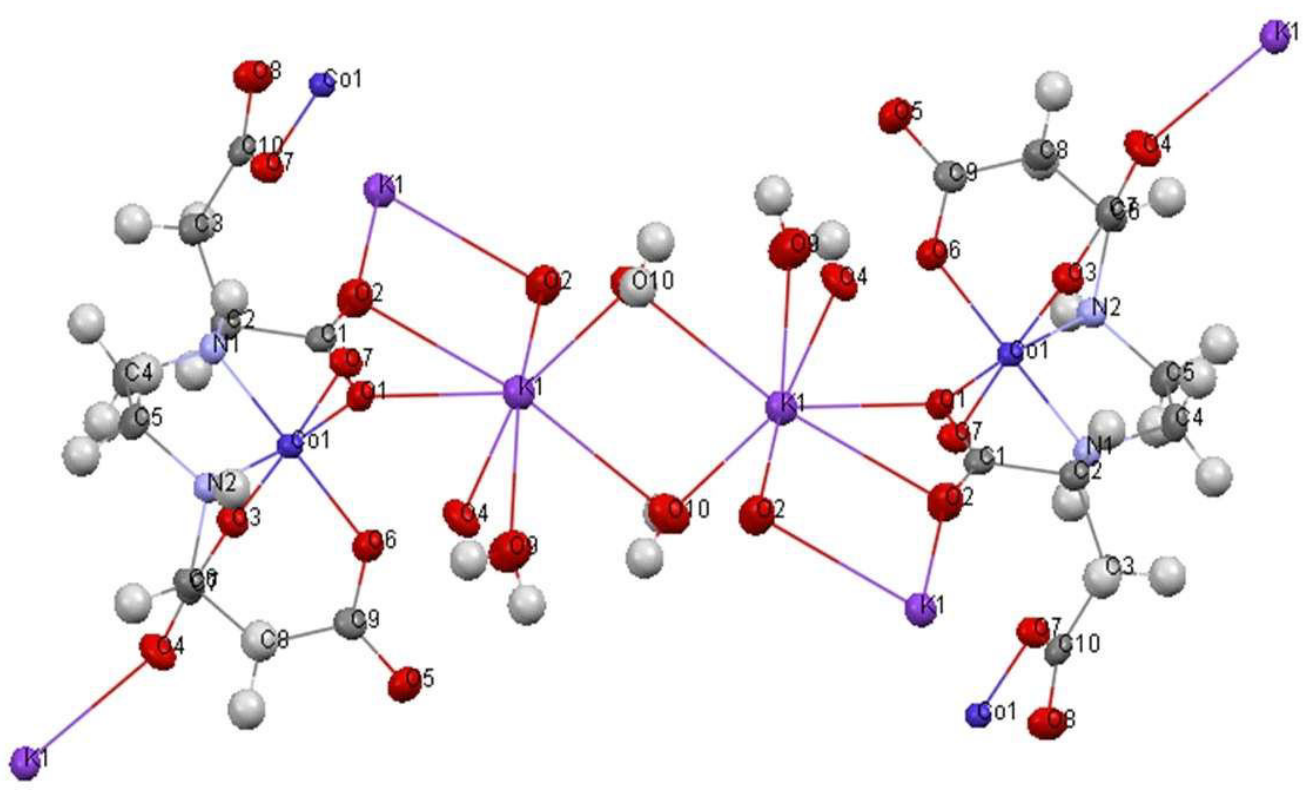

Fig. 2. Molecular structure of the complex under study 


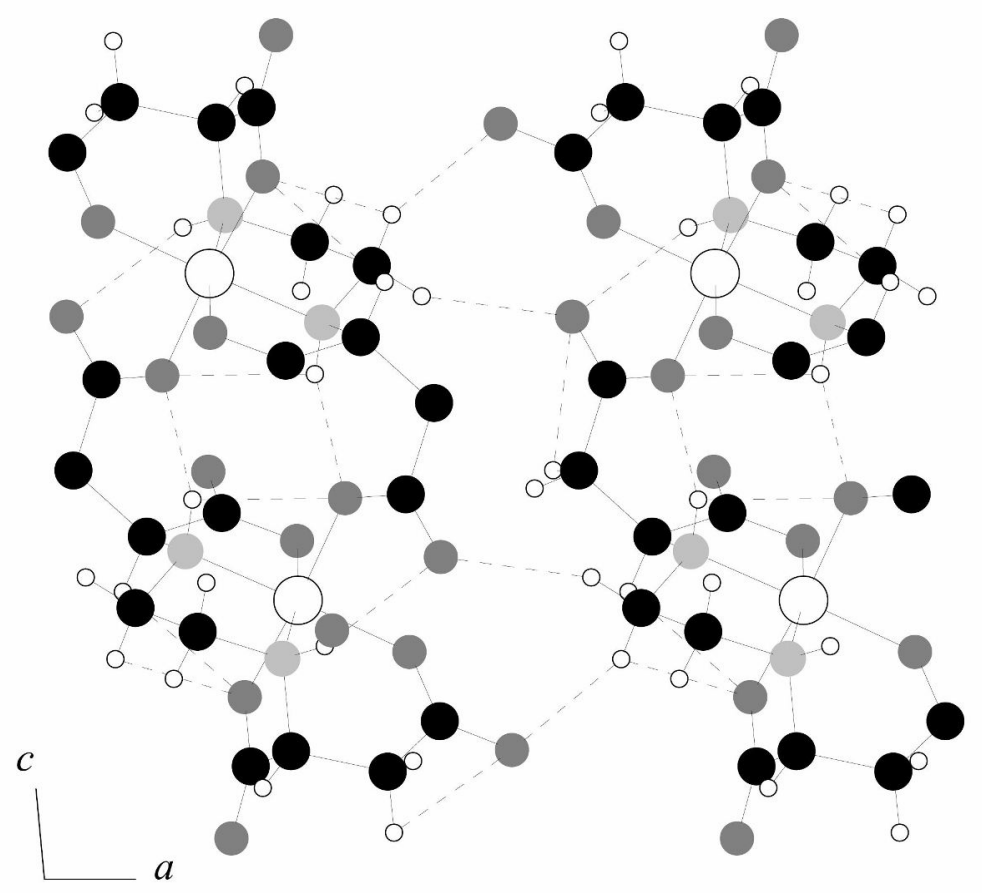

Fig. 3. Hydrogen bonding of two adjacent $\left[\left(\mathrm{C}_{10} \mathrm{H}_{12} \mathrm{~N}_{2} \mathrm{O}_{8}\right)_{2} \mathrm{Co}_{2}\right]$ moieties viewed along the [010] direction. Color codes: light grey nitrogen, dark grey - oxygen, black-filled spheres are carbon atoms. Empty spheres indicate hydrogen, cobalt and potassium atoms are of sizes proportional to their covalent radii

Table 4

Geometric parameters of hydrogen bonds

\begin{tabular}{c|c|c|c|c}
\hline $\mathrm{D}-\mathrm{H} \cdots \mathrm{A}$ & $\mathrm{d}(\mathrm{D}-\mathrm{H}), \AA$ & $\mathrm{d}(\mathrm{H} \cdots \mathrm{A}), \AA$ & $\mathrm{d}(\mathrm{D} \cdots \mathrm{A}), \AA$ & $\theta(\mathrm{D}-\mathrm{H} \cdots \mathrm{A}),^{\circ}$ \\
\hline $\mathrm{N} 2-\mathrm{H} 5 \cdots \mathrm{O} 4^{\text {vii }}$ & 0.90 & 2.40 & $3.0920(18)$ & 134 \\
\hline $\mathrm{N} 2-\mathrm{H} 5 \cdots 8^{\mathrm{i}}$ & 0.90 & 2.26 & $2.9520(18)$ & 134 \\
\hline $\mathrm{N} 1-\mathrm{H} 10 \cdots \mathrm{O} 7$ & 0.87 & 2.04 & $2.7490(17)$ & 138 \\
\hline $\mathrm{O} 9-\mathrm{H} 14 \cdots \mathrm{O} 6^{\mathrm{vi}}$ & 0.74 & 2.22 & $2.9311(18)$ & 159 \\
\hline $\mathrm{O} 9-\mathrm{H} 15 \cdots \mathrm{O} 8^{\mathrm{iii}}$ & 0.89 & 1.85 & $2.7385(17)$ & 174 \\
\hline $\mathrm{O} 10-\mathrm{H} 16 \cdots \mathrm{O} 5$ & 0.88 & 2.03 & $2.8766(17)$ & 160 \\
\hline $\mathrm{O} 10-\mathrm{H} 17 \cdots \mathrm{O} 6^{\mathrm{x}}$ & 0.87 & 2.22 & $3.0162(18)$ & 152 \\
\hline $\mathrm{C} 5-\mathrm{H} 4 \cdots \mathrm{O}^{\mathrm{xi}}$ & 0.98 & 2.59 & $3.464(2)$ & 148 \\
\hline $\mathrm{C} 5-\mathrm{H} 4 \cdots \mathrm{O} 3^{\text {viii }}$ & 0.98 & 2.48 & $3.028(2)$ & 115 \\
\hline $\mathrm{C} 3-\mathrm{H} 8 \cdots \mathrm{O} 3$ & 0.97 & 2.56 & $2.928(2)$ & 103 \\
\hline $\mathrm{C} 3-\mathrm{H} 8 \cdots \mathrm{O} 6^{\text {xii }}$ & 0.97 & 2.33 & $3.222(2)$ & 152 \\
\hline $\mathrm{C} 3-\mathrm{H} 9 \cdots \mathrm{O} 8^{\mathrm{v}}$ & 0.97 & 2.44 & $3.350(2)$ & 157 \\
\hline $\mathrm{C} 2-\mathrm{H} 13 \cdots \mathrm{O} 3$ & 0.98 & 2.56 & $2.923(2)$ & 102 \\
\hline
\end{tabular}

branches of ligand are replaced in the axial position of the distorted $\mathrm{Co}(\mathrm{II})$ octahedron. The combination of ethylenediamine (with angle $\mathrm{N}-\mathrm{Co}-\mathrm{N}<90^{\circ}$ ) and glycine (with angle $\mathrm{N}-\mathrm{Co}-\mathrm{O} \geq 90^{\circ}$ ) cycles decreases the distortion of cobalt octahedron in the equatorial plane compared to the sodium-containing complex.

Acknowledgments

The authors thank PhD Anatoliy Dudko for his assistance in performing X-ray diffraction analysis.

\section{REFERENCES}

1. Biodegradation and speciation of residual SSethylenediaminedisuccinic acid (EDDS) in soil solution left after soil washing / Tandy S., Ammann A., Schulin R., Nowack B. // Environmental Pollution. - 2006. - Vol.142. - P.191-199.

2. Effect of accompanying ions and ethylenediaminedisuccinic acid on heavy metals sorption using hybrid materials Lewatit FO 36 and Purolite Arsen $\mathrm{X}^{\mathrm{np}} /$ 


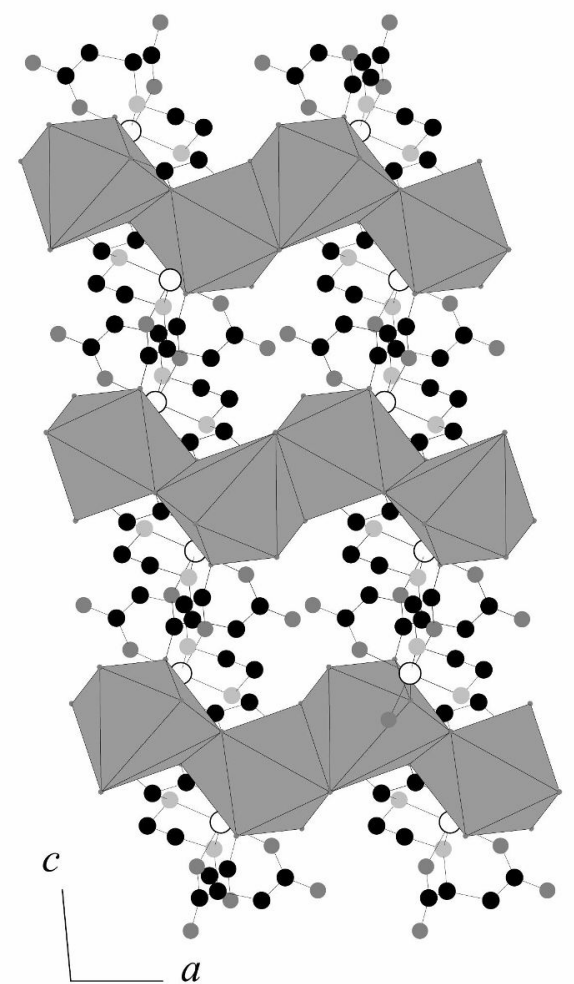

Fig. 4. Extension of the $\left[\mathrm{KO}_{7}\right]$ polyhedrons (grey-filled on the picture) along the [001] direction

Kolodynska D., Kowalczyk M., Hubicki Z., et al. // Chemical Engineering Journal. - 2015. - Vol.276. - P.376-387.

3. Removal of copper, iron and zinc from soil washing effluents containing ethylenediaminedisuccinic acid as chelating agent through sunlight driven nano- $\mathrm{TiO}_{2}$-based photocatalytic processes / Clarizia L., Race M., Onotri L., et al. // Nanotechnologies for Environmental Remediation. - Springer, Cham. - 2017. - P.239-253.

4. Jones P.W., Williams D.R. Speciation efficiency indices (SEI) and readily-biodegradable indices (RBI) for optimising ligand control of environmental and associated industrial processes // International Journal of Environmental Analytical Chemistry. - 2001. - Vol.81. - P.73-88.

5. Шадчина Т.М., Прядкина Г.О., Трунова О.К. Антихлорозні властивості комплексонатів мікроелементів (заліза та міді) // Физиология и биохимия культурных растений. 2008. - T.40. - C.435-440.

6. Biodegradable chelating agent ethylenediaminedisuccinic acid reduces uptake of copper through alleviation of copper toxicity in hydroponically grown Chrysanthemum Coronarium L. / Wei L., Luo Ch., Wang Ch., et al. // Environmental Toxicology and Chemistry. - 2007. - Vol.26. - P.749-754.

7. Pavelcik F., Majer J. The crystal and molecular structure of lithium [(S,S)-N,N'-ethylenediaminedisuccinato]cobaltate(III) trihydrate // Acta Crystallographica Section B. - 1978. - Vol.34. - P.3582-3585.

8. Бария бис(этилендиаминодисукцинато-кобальт(III)) гептагидрат / Засурская Л.А., Поленова Т.Н., Письменская Е.Б., Позняк А.Л. // Кристаллография. - 1997. - Том.42. - С.107-111.

9. Organic-soluble optically pure anionic metal complexes $\mathrm{PPh}_{4}\left[\mathrm{M}^{\mathrm{II}}(\mathrm{S}, \mathrm{S}-\mathrm{EDDS})\right] \cdot 2 \mathrm{H}_{2} \mathrm{O}(\mathrm{M}=\mathrm{Fe}, \mathrm{Co}, \mathrm{Cr}) / \mathrm{Chmel}$ N.P., Howson S.E., Allan L.E.N., et al. // Dalton Transactions. 2010. - Vol.39. - P.2919-2927.

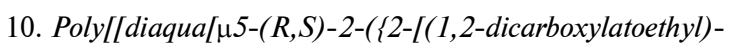
amino]ethyl\}amino)butanedioato]cobaltate(III)sodium]dihydrate] / Trunova O.K., Dudko A.V., Makotryk T.O., et al. // Acta Crystallographica Section E. - 2011. - Vol.67. - P.1547-1548.

11. Технологические основы синтеза биологически активного комплексона на основе янтарной кислоты / Трунова Е.К., Мазуренко Е.А., Макотрик Т.А., Роговцов А.А. // Вопр. химии и хим. технологии. - 2001. - № 4. - С.28-31.

12. Sheldrick G.M. A short history of SHELX // Acta Crystallographica Section A. - 2008. - Vol.64. - P.112-122.

13. Wood R.M., Palenik G.J. Bond valence sums in coordination chemistry. A simple method for calculating the oxidation state of cobalt in complexes containing only $\mathrm{Co}-\mathrm{O}$ bonds // Inorganic Chemistry. - 1998. - Vol.37. - P.4149-4151.

14. Cremer D., Pople J.A. General definition of ring puckering coordinates // Journal of the American Chemical Society. - 1975. - Vol.97. - P.1354-1358.

Received 16.10.2018

\section{КРИСТАЛІЧНА БУДОВА НОВОГО КОМПЛЕКСУ КОБАЛЬТУ(ІІ) 3 ЕТИЛЕНДИАМІНДИ ЯНТАРНОЮ КИСЛОТОЮ}

О.К. Трунова, А.М. Міщенко, О.О. Роговщов, Т.О. Макотрик

Завдяки своїм цінним властивостям координаційні сполуки $3 d$ перехідних металів з амінокарбоновими кислотами $\epsilon$ перспективними біологічно активними сполуками. В даній роботі синтезовано дигідрат (етилендиамін-N,N'-дисукиинато)кобальтату (II) калію і досліджено його будову методом рентгеноструктурного аналізу. Визначення структури виконано шляхом аналізу найбільш інтенсивних 6669 відбиттів, структуру уточнено методом найменших квадратів. Дана сполука являє собою біядерний комплекс Со(II) з етилендиаміндибурштинною кислотою закритого типу, що складається з фрагментів $\left[\left(\mathrm{C}_{10} \mathrm{H}_{12} \mathrm{~N}_{2} \mathrm{O}_{8}\right)_{2} \mathrm{Co}_{2}\right]$, сполучених між собою атомами калію з утворенням зигзагоподібної структури, $і$ належить до просторової групи $P 2 /$. Кожен з іонів Со(II) знаходиться у викривленому октаедричному оточенні, утвореному трьома 5-членними та одним 6-членним хелатними циклами. Одну $з$ позицій в октаедрі $\left[\mathrm{CoO}_{4} \mathrm{~N}_{2}\right]$ займає атом кисню сусідньої молекули ліганду. При цьому ацетатні гілки лігандів розташовані в аксіальних позиціях октаедру атома Со(II), на відміну від раніше дослідженого натрієвмісного комплексу аналогічного складу. Координаційна сфера атома калію утворена трьома атомами кисню молекул води, двома атомами кисню сусідньої карбоксильної групи, а також двома атомами кисню двох сусідніх молекул ліганду. Досліджений комплекс також демонструє розгалужену систему міжлігандних $N-H \cdots O, C-H \cdots O$ та гідратних $O-H \cdots O$ водневих зв'язків.

Ключові слова: комплекс кобальту, етилендиаміндибурштинна кислота, рентгеноструктурний аналіз, хелатний цикл, водневий зв'язок. 


\section{CRYSTAL STRUCTURE OF A NOVEL COBALT(II) COMPLEX WITH ETHYLENEDIAMINEDISUCCINIC ACID}

E.K. Trunova, A.M. Mishchenko, A.A. Rogovtsov, T.A. Makotryk V.I. Vernadsky Institute of General and Inorganic Chemistry of the NAS of Ukraine, Kyiv, Ukraine

Due to their valuable properties, coordination compounds of $3 d$ transition metals with aminocarboxylic acids are perspective bioactive compounds. In the present work, potassium (ethylenediamine- $N, N$ '-disuccinato)cobaltate(II) dihydrate was prepared and structurally characterized by single-crystal $X$-ray diffraction analysis. Structure determination was performed by a least-squares fit of 6669 reflections of highest intensity and refined with the full-matrix least squares procedure. Present compound is a closed-type binuclear Co(II) complex of ethylenediaminedisuccinic acid composed of $\left[\left(\mathrm{C}_{10} \mathrm{H}_{12} \mathrm{~N}_{2} \mathrm{O}_{8}\right)_{2} \mathrm{Co}_{2}\right]$ moieties bridged via potassium atoms in a zig-zag fashion and possesses the monoclinic $P 2_{1} / c$ space group. Each $\mathrm{Co}($ II) ion has a distorted octahedral environment formed by sets of three 5-membered and one 6-membered chelate cycles. One of the positions in the $\left[\mathrm{CoO}_{4} \mathrm{~N}_{2}\right]$ octahedron is occupied by oxygen atom of the neighboring ligand molecule. The acetate branches of ligand are situated in the axial positions of the distorted Co(II) octahedron on the contrary to previously studied sodium-containing complex of analogous composition. Each potassium atom has a sevenfold coordination involving three oxygen atoms of water molecules, two oxygen atoms from the nearest carboxyl group and two additional oxygen atoms from two adjacent ligand molecules. The complex under study also displays a developed system of inter-ligand $\mathrm{N}-\mathrm{H} \cdots \mathrm{O}$, $\mathrm{C}-\mathrm{H} \cdots \mathrm{O}$ and hydration $\mathrm{O}-\mathrm{H} \cdots \mathrm{O}$ bonds.

Keywords: cobalt complex; ethylenediaminedisuccinic acid; $\mathrm{X}$-ray structure analysis; chelate cycle; hydrogen bond.

\section{REFERENCES}

1. Tandy S., Ammann A., Schulin R., Nowack B. Biodegradation and speciation of residual $\mathrm{SS}$ ethylenediaminedisuccinic acid (EDDS) in soil solution left after soil washing. Environmental Pollution, 2006, vol. 142, pp. 191199.

2. Kolodynska D., Kowalczyk M., Hubicki Z., Shvets V., Golub V. Effect of accompanying ions and ethylenediaminedisuccinic acid on heavy metals sorption using hybrid materials Lewatit FO 36 and Purolite Arsen $\mathrm{X}^{\mathrm{np}}$. Chemical Engineering Journal, 2015, vol. 276, pp. 376-387.

3. Clarizia L., Race M., Onotri L., Di Somma I., Fiorentino N., Andreozzi R., Marotta R. Removal of copper, iron and zinc from soil washing effluents containing ethylenediaminedisuccinic acid as chelating agent through sunlight driven nano- $\mathrm{TiO}_{2}$-based photocatalytic processes. In: Lofrano G., Libralato G., Brown J. (Eds.). Nanotechnologies for Environmental Remediation. Springer, Cham, 2017, pp. 239-253.
4. Jones P.W., Williams D.R. Speciation efficiency indices (SEI) and readily-biodegradable indices (RBI) for optimising ligand control of environmental and associated industrial processes. International Journal of Environmental Analytical Chemistry, 2001, vol. 81 , pp. 73-88.

5. Shadchina T.M., Pryadkina G.O., Trunova, O.K. Antykhlorozni vlastyvosti kompleksonativ mikroelementiv (zaliza ta midi) [Antichloric properties of microelement complexonates]. Physiology and Biochemistry of Cultivated Plants, 2008, vol. 40, pp. 435-440. (in Ukrainian).

6. Wei L., Luo C., Wang C., Li X., Shen Z. Biodegradable chelating agent ethylenediaminedisuccinic acid reduces uptake of copper through alleviation of copper toxicity in hydroponically grown Chrysanthemum Coronarium L. Environmental Toxicology and Chemistry, 2007, vol. 26, pp. 749-754.

7. Pavelcik F., Majer J. The crystal and molecular structure of lithium [(S,S)-N,N'-ethylenediaminedisuccinato]cobaltate(III) trihydrate. Acta Crystallographica Section B, 1978, vol. 34, pp. 3582-3585.

8. Zasurskaya L.A., Polynova T.N., Pis'menskaya E.B., Poznyak A.L. Bariya bis(etilendiaminodisuktsinato-kobalt(III) geptagidrat [Barium bis(ethylenediaminodisuccinato-cobalt(III)) heptahydrate]. Crystallography Report, 1997, vol. 42, pp. 107111. (in Russian).

9. Chmel N.P., Howson S.E., Allan L.E.N., Barker J., Clarkson G.J., Turner S.S., Scott P. Organic-soluble optically pure anionic metal complexes $\mathrm{PPh}_{4}\left[\mathrm{M}^{\mathrm{III}}(\mathrm{S}, \mathrm{S}-\mathrm{EDDS})\right] \cdot 2 \mathrm{H}_{2} \mathrm{O}(\mathrm{M}$ $=\mathrm{Fe}, \mathrm{Co}, \mathrm{Cr})$. Dalton Transactions, 2010, vol. 39, pp. 29192927.

10. Trunova O.K., Dudko A.V., Makotryk T.O., Osadcha O.V., Pekhnyo V.I., Shovkova G.V. Poly[[diaqua[ $\mu 5-(R, S)-2-$ (\{2-[(1,2-dicarboxylatoethyl)amino]ethyl\}amino)butanedioato]cobaltate(III)sodium]dihydrate]. Acta Crystallographica Section E, 2011, vol. 67, pp. m1547-m1548.

11. Trunova E.K., Mazurenko E.A., Makotrik T.A., Rogovtsov A.A. Tekhnologicheskie osnovy sinteza biologicheski aktivnogo kompleksona na osnove yantarnoi kisloty [Technological aspects of synthesis of bioactive complexone based on succinic acid]. Voprosy Khimii i Khimicheskoi Tekhnologii, 2001, no. 4, pp. 28-31. (in Russian).

12. Sheldrick G.M. A short history of SHELX. Acta Crystallographica Section A, 2008, vol. 64, pp. 112-122.

13. Wood R.M., Palenik G.J. Bond valence sums in coordination chemistry. A simple method for calculating the oxidation state of cobalt in complexes containing only $\mathrm{Co}^{-} \mathrm{O}$ bonds. Inorganic Chemistry, 1998, vol. 37, pp. 4149-4151.

14. Cremer D., Pople J.A. General definition of ring puckering coordinates. Journal of the American Chemical Society, 1975, vol. 97, pp. 1354-1358. 